\title{
Diabetes gestacional na perspectiva de mulheres grávidas hospitalizadas
}

\author{
Gestational diabetes from the perspective of hospitalized pregnant women \\ Diabetes gestacional desde la perspectiva de mujeres embarazadas hospitalizadas
}

\author{
Márcio Flávio Moura Araújo', Sarah Maria Fraxe Pessoa", \\ Marta Maria Coelho Damasceno"II, Maria Lúcia Zanetti ${ }^{\text {IV }}$ \\ ' Universidade Federal do Ceará, Programa de Pós-Graduação em Enfermagem (Doutorando). Fortaleza-CE, Brasil. \\ "Universidade Federal do Ceará, Maternidade Escola Assis Chateaubriand. Fortaleza-CE, Brasil. \\ II' Universidade Federal do Ceará, Faculdade de Farmácia Odontologia e Enfermagem, \\ Programa de Pós-Graduação em Enfermagem. Fortaleza-CE, Brasil. \\ IV Universidade de São Paulo, Escola de Enfermagem de Ribeirão Preto, \\ Departamento de Enfermagem Geral e Especializada. Ribeirão Preto-SP, Brasil.
}

Submissão: 06-07-2011 Aprovação: 10-04-2013

RESUMO

Estudo qualitativo fenomenológico, que teve como objetivo compreender o significado das experiências vivenciadas por mulheres com diabetes mellitus gestacional. Participaram 12 pacientes internadas em uma maternidade de Fortaleza-CE, Brasil, que manifestaram seus sentimentos e percepções por meio de entrevista aberta e desenhos. O material empírico foi transcrito na íntegra e, posteriormente, organizado e analisado pelo método fenomenológico. Os resultados evidenciaram dois temas: (1) Vivenciando experiências que trazem felicidade e bem-estar e (2) Vivenciando experiências que provocam sofrimento. Este estudo fenomenológico, ao possibilitar apreender a experiência das mulheres com diabetes mellitus gestacional, contribui para o planejamento e a implementação de programas de intervenção baseados em um modelo de saúde participativo, com vistas a priorizar os aspectos subjetivos envolvidos na gravidez de risco.

Descritores: Diabetes Gestacional; Complicações na Gravidez; Saúde Materno-Infantil; Cuidados de Enfermagem; Pesquisa Qualitativa.

\section{ABSTRACT}

This phenomenological qualitative study aimed to understand the meaning of the experiences lived by women with gestational diabetes mellitus. The sample consisted of 12 patients hospitalized at a maternity hospital in the city of Fortaleza, state of Ceará, Brazil, which expressed their feelings and perceptions through open interviews and drawings. The empirical material was fully transcribed and then organized and analyzed by the phenomenological method. The results revealed two themes: (1) Living experiences that bring happiness and well-being, and (2) Living experiences that cause suffering. This phenomenological study showed the experience of women with gestational diabetes mellitus, thus enabling to plan and to implement intervention programs based on a participatory model of health in order to prioritize the subjective aspects involved in high-risk pregnancy. Key words: Diabetes Gestational; Pregnancy Complications; Maternal and Child Health; Nursing Care; Qualitative Research.

\section{RESUMEN}

Estudio fenomenológico cualitativo que objetivó comprender el significado de las experiencias vividas por las mujeres con diabetes mellitus gestacional. Los participantes fueron 12 pacientes ingresadas en un hospital de maternidad en la ciudad de Fortaleza, estado del Ceará, Brasil, que expresaran sus sentimientos y percepciones a través de entrevistas abiertas y dibujos. El material empírico fue transcrito completamente y luego organizado y analizado por el método fenomenológico. Los resultados revelaron dos temas: (1) Viviendo experiencias que traen felicidad y bienestar y (2) Viviendo experiencias que causan sufrimiento. Este estudio fenomenológico, que mostró la experiencia de las mujeres con diabetes mellitus gestacional, contribuye a la planificación y ejecución de programas de intervención basados en un modelo participativo de la salud, con el fin de dar prioridad a los aspectos subjetivos que intervienen en embarazos de riesgo.

Palabras clave: Diabetes Gestacional; Complicaciones del Embarazo; Salud Materno-Infantil; Atención de Enfermería; Investigación Cualitativa. 


\section{INTRODUÇÃO}

O diabetes mellitus gestacional (DMG) é o problema metabólico mais comum na gestação. Tem prevalência entre $3 \%$ a $13 \%$ das gravidezes, dependendo de cor/raça, população e critérios diagnósticos utilizados ${ }^{(1)}$. Caracteriza-se por qualquer nível de intolerância a carboidratos, que resulta em hiperglicemia de intensidade variável, identificada pela primeira vez no decorrer da gestação ${ }^{(2-3)}$. O DMG vem crescendo em proporções semelhantes à prevalência do diabetes mellitus tipo 2 (DM2). Nos últimos 20 anos, aumentou significativamente nos Estados Unidos, onde foi registrada prevalência de $1,4 \%$ a $6,1 \%$, enquanto que, no Brasil, estima-se estar entre $2,4 \%$ e $7,2 \%{ }^{(1,3-4)}$.

O controle inadequado do DMG aumenta os riscos, as complicações e os efeitos adversos para o binômio mãe-filho no período pré-natal e neonatal. Ter diabetes na gestação eleva a possibilidade de desenvolvimento de DM2 para a muIher após o parto e, em cada gravidez, aumenta o risco das crianças desenvolverem a doença ${ }^{(5)}$. Esses aspectos advindos da confirmação do diagnóstico, por sua vez, desencadeiam sérias repercussões no estado emocional das gestantes acometidas ${ }^{(6)}$, agravadas pela obrigatoriedade da internação.

Dadas as variadas facetas que envolvem o assunto em pauta, ultimamente o DMG tem sido amplamente estudado em seus aspectos fisiopatológicos, com destaque para a busca de evidências fortes que possam ser utilizadas na prática clínica ${ }^{(1,7-8)}$. Entretanto, pouco se conhece sobre as experiências vivenciadas pelas gestantes que desenvolvem o DMG, o que evidencia a existência de lacunas na literatura acerca desse conhecimento específico ${ }^{(5)}$. Particularmente, no que se refere à cidade de Fortaleza - Ceará, as experiências das mulheres acerca do diabetes mellitus na gravidez ainda não foram investigadas. Desse modo, o objetivo da presente pesquisa foi compreender o significado das experiências vivenciadas por mulheres com DMG.

\section{MÉTODO}

Trata-se de um estudo qualitativo. O pesquisador que utiliza essa abordagem acredita que os seres humanos são únicos e atribuem significados singulares às suas experiências e que essas derivam do seu contexto de vida, isto é, da matriz de relações estabelecidas entre os seres humanos versus ambiente, que constituem o cotidiano ${ }^{(9)}$. O referencial teórico-metodológico adotado foi a Fenomenologia, que permite descrever em profundidade a experiência humana e construir seu significado por meio do diálogo intensivo, neste caso, com mulheres acometidas pelo $\mathrm{DMG}^{(10)}$.

A investigação foi desenvolvida na clínica obstétrica de uma maternidade-escola situada em Fortaleza, no período de abril a outubro de 2007. Foram recrutadas, por conveniência, 12 mulheres mediante convite feito durante visita às enfermarias. Estabeleceram-se os critérios de inclusão: ter diagnóstico de DMG confirmado no prontuário de saúde e estar internada no período da coleta de dados. Como critérios de exclusão foram considerados: gestantes que tinham outras condições clínicas associadas, bem como as que estavam impossibilitadas de participar das sessões de arteterapia.
As participantes assinaram o Termo de Consentimento Livre e Esclarecido e as entrevistas abertas foram iniciadas com a questão: Conte-me como é, para você, ter diabetes gestacional e estar internada?

Adicionalmente, para obter dados em profundidade, foram elaboradas questões complementares: Fale sobre o que você sentiu quando soube que tinha diabetes gestacional; Fale sobre o que sentiu quando soube que iria ficar internada; Como tem sido seu relacionamento com a família no período da internação? Como tem sido seu relacionamento com os profissionais durante a internação? Como tem sido seu relacionamento com as outras pacientes da enfermaria? Como você está enfrentando esta situação?

Também foi solicitado que as participantes expressassem, por meio de desenhos, os sentimentos que emergiram da vivência do fenômeno investigado. O desenho é uma forma de linguagem que permite à pessoa comunicar-se com os outros, possibilitando ao ser humano manifestar ideias, crenças e sentimentos ao se defrontar com determinadas situações ${ }^{(11)}$.

Além disso, utilizou-se um formulário para o registro dos dados sociodemográficos, do histórico obstétrico e da gestação atual, os quais foram obtidos por meio da consulta ao prontuário de saúde.

A coleta de dados ocorreu nas enfermarias e as entrevistas foram audiogravadas, bem como os relatos verbais produzidos pelas participantes a partir de seus desenhos. A amostra foi definida a partir da saturação dos discursos ${ }^{(12)}$.

O material empírico foi transcrito na íntegra e, posteriormente, organizado e analisado pelo método fenomenológico, tal como proposto por Colaizzi ${ }^{(13)}$, que abrange os passos seguintes: (1) ler todos os dados para visualizar o todo; (2) revisar cada dado e extrair as afirmações significativas; (3) declarar o significado de cada afirmação significativa, formular significados; (4) organizar os significados formulados em grupos de temas; (5) integrar os resultados em uma descrição exaustiva do fenômeno em estudo; (6) formular uma descrição exaustiva do fenômeno em estudo, com uma afirmação de identificação tão inequívoca quanto possível; (7) questionar os participantes sobre os achados, como um passo final de validação. No presente estudo, os resultados também foram validados com as enfermeiras do serviço.

Ademais, utilizou-se a literatura pertinente ao tema e os conceitos da Fenomenologia Existencial de Heidegger ${ }^{(14)}$. Vale destacar que os desenhos foram interpretados pelas próprias participantes, uma vez que foram utilizados seus relatos - e não os desenhos em si - como fonte de acesso às vivências, complementar às entrevistas.

O projeto de pesquisa foi aprovado pelo Comitê de Ética em Pesquisa da Maternidade Escola Assis Chateaubriand, Protocolo $n^{\circ} 03 / 2007$. A pesquisa foi conduzida em conformidade com os padrões éticos exigidos.

\section{RESULTADOS}

As doze participantes tinham entre 19 e 44 anos; onze eram casadas, quatro haviam cursado o Ensino Médio e três, o Fundamental. Quatro eram empregadas domésticas e três, 
do lar. Quatro estavam na primeira gestação; três, na segunda e outras três, na terceira.

O corpus de pesquisa foi constituído pelo conjunto formado pelas entrevistas e relatos produzidos sobre os desenhos. Da análise empreendida, emergiram dois temas: "vivenciando experiências que trazem felicidade e bem-estar" e "vivenciando experiências que provocam sofrimento".

\section{A. Vivenciando experiências que trazem felicidade e bem-estar}

Apesar de se encontrarem em situação de risco e distantes da família e de seus domicílios, as participantes expressaram sentimentos prazerosos diversificados, que foram divididos em três subtemas: possibilidade de ser mãe, acesso à internação hospitalar e convivência com outras mulheres sob condições idênticas.

\section{Possibilidade de ser mãe}

Só de lembrar que estou com uma criança, uma criança que eu desejei muito [...]. (P1)

\section{Estar grávida para mim é uma realização [...]. (P2)}

Algumas mulheres tinham histórico de abortos porque desenvolveram DMG em gravidezes anteriores. A alegria pela nova oportunidade de ter filhos, bem como a esperança de levar a gestação a termo e ter uma criança saudável, permeavam seu cotidiano. Ao complementar a descrição de sua vivência por meio do desenho (Figura 1), uma das participantes expressou que, apesar de tudo, ainda tinha muitos motivos para comemorar.

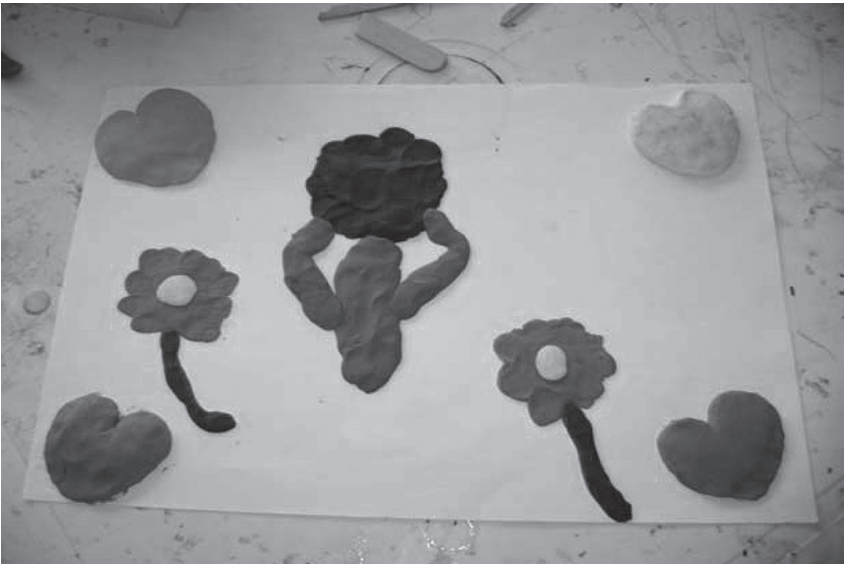

Figura 1 - Nesse desenho tem corações, árvores e flores que, juntos, formam um bonito quadro representando a minha felicidade [...]. (P3)

\section{Acesso à internação hospitalar}

Quase todas as mulheres relataram que tiveram fácil acesso à hospitalização, o que possibilita um controle rigoroso do DMG em virtude dos cuidados da equipe de profissionais de saúde. Essa acessibilidade despertou nas gestantes a esperança da cura após o parto, fortalecendo o sentimento de segurança e a motivação para o autocuidado. Assim, ao superarem o impacto do diagnóstico, começaram a lutar, sobretudo pela saúde do bebê.

[...] mas, depois que eu soube que poderia ser tudo recuperado, eu me conformei, parti para a batalha [...] eu vou ter vitória [...]. (P3)

Eu estar aqui, recebendo todo esse tratamento, está me dando força [...] eu vou ter minha filha sadia [...]. (P1)

O nascimento de um bebê saudável foi uma preocupação recorrente manifestada pelas grávidas e, por esse motivo, referiram suportar bem a hospitalização. Uma das gestantes destacou que se considerava feliz porque, após enfrentar dificuldades para obter a internação em outras instituições de saúde, finalmente conseguiu ser hospitalizada para o tratamento do DMG, minimizando assim seu sofrimento.

[...] eu tive dificuldade de ser recebida nos outros hospitais [...] fiquei abalada quando me mandaram de volta [...] quando me receberam aqui foi uma vitória. Eu estou feliz por estar aqui [...]. (P1)

Os sentimentos que emergiram desse discurso também foram manifestados por meio do desenho (Figura 2), que ilustra a transformação da tristeza em alegria.

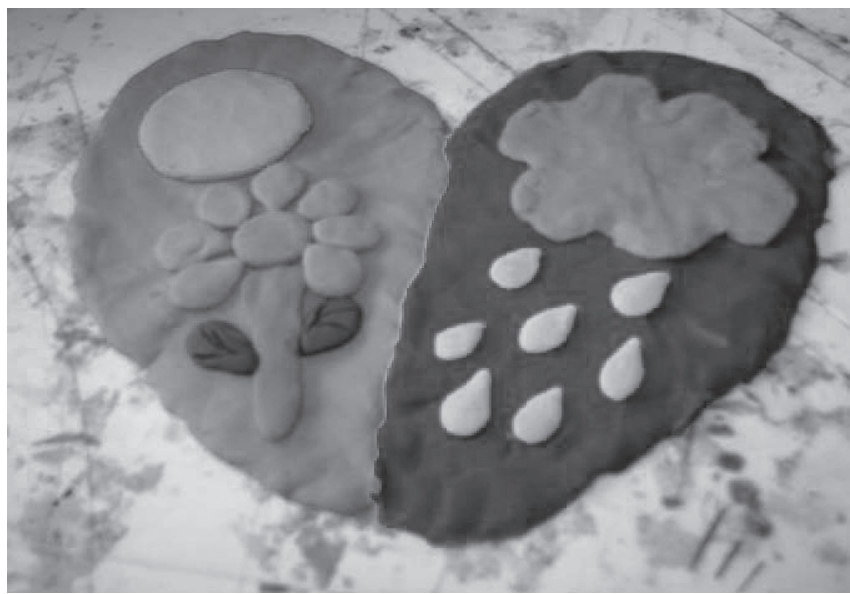

Figura 2 - Os dias de chuva representam a tristeza pela dificuldade de acesso à hospitalização, e os dias de sol, a alegria de ter iniciado o tratamento. (P1)

A relação com os profissionais da clínica obstétrica também foi descrita como um recurso importante para obter bons resultados no tratamento. As participantes destacaram a dedicação da equipe de saúde, o que está retratado nos trechos seguintes. 
A minha médica e outras pessoas que vêm para me acompanhar têm uma atenção muito grande [...] As enfermeiras gostam de brincar, para apagar aquela coisa de momento ruim, quando vem aquela furadinha [...]. (P1)

Aqui me trataram bem, são muito educados [...] Agora estou mais calma, já consegui controlar a pressão e a glicose [...]. (P4)

Convivência com outras mulheres em condições idênticas

Compartilhar com outras pessoas o mesmo diagnóstico foi percebido pelas gestantes como uma experiência positiva, configurando uma rede de apoio que se inicia e consolida-se durante a internação. As novas relações cotidianas, estabelecidas com as demais gestantes, evidenciam a possibilidade de olhar o outro que está em situação idêntica ou até mais grave. Assim, a solidariedade se estabelece na relação de um ser com o outro, dando lugar a uma interação prazerosa, caracterizada pelo cuidado consigo e com o outro.

Porque a gente vai ouvindo, vai convivendo com as pessoas, e a gente vê que não é só a gente que está com problemas [...] então, eu estou procurando encarar que o meu problema, perto de muitas, é até pequeno [...]. (P5)

E você pode, apesar da sua dificuldade, ajudar alguém, e eu estou ajudando... é uma lição de vida. (P6)

Os benefícios advindos das relações interpessoais deflagradas a partir da internação foram ilustrados na Figura 3. No quadrante superior esquerdo da figura estão representadas as colegas de enfermaria da participante e o bom relacionamento estabelecido com elas.

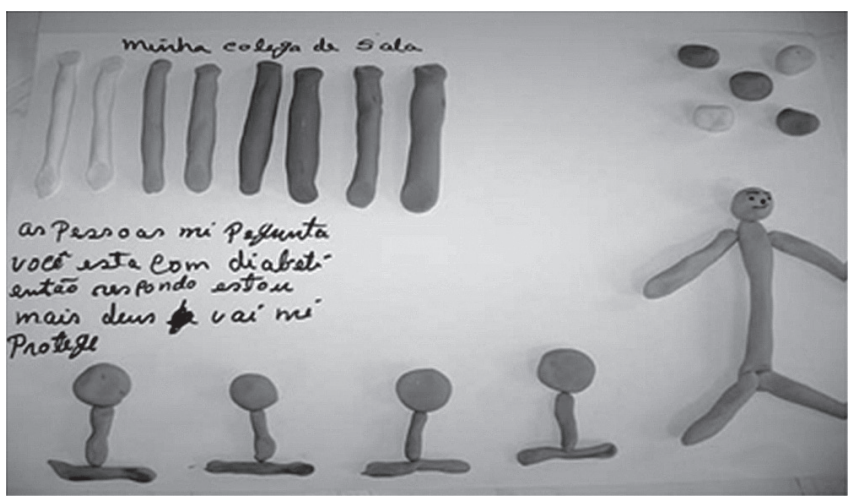

Figura 3 - A boneca sou eu e os cogumelos são para enfeitar. Minha vida depois do diabetes tem sido aqui no hospital. (P7)

\section{B. Vivenciando experiências que provocam sofrimento}

Além dos aspectos positivos vivenciados com o DMG, as participantes expressaram sentimentos que sugerem sofrimento. Essas vivências foram subdivididas em dois subtemas: impacto do diagnóstico, afastamento da família e do domicílio.

\section{Impacto do diagnóstico}

O impacto do diagnóstico inesperado de DMG ocasionou temores devido a suas possíveis consequências para as gestantes e os bebês, bem como a necessidade de internação. Uma das participantes relatou que veio sozinha para a consulta pré-natal, como fazia todos os meses, e que, tendo sido comunicada sobre a necessidade da internação imediata, não pôde retornar para casa para se preparar.

Eu vim sozinha para fazer uma consulta pré-natal [...] foi um choque [...]. (P2)

[...] a gente corre muito risco [...] tenho medo que alguma coisa aconteça com a minha filha [...]. (P7)

O temor manifestado também esteve relacionado a experiências anteriores de perdas de familiares em decorrência de complicações do DM.

\section{Meu pai teve diabetes e faleceu devido a isso. (P8)}

A figura 4 representa, segundo a descrição da participante, a reação emocional frente ao impacto do recebimento abrupto do diagnóstico.



Figura 4 - [...] Quando eu descobri que estava com diabetes o meu coração ficou assim, bem apertado, e eu me senti presa, sem saber o que fazer, para onde correr [...] O rosto é o meu rosto triste, preocupada [...]. (P2)

\section{Afastamento em relação à família e ao domicílio}

A ruptura da sensação de continuidade do cotidiano e o distanciamento em relação aos familiares e o lar provocam, inicialmente, a não aceitação da situação crítica vivenciada, sobretudo quando estão envolvidos filhos menores, dependentes de cuidados. Em alguns casos, as participantes contam com uma rede social de apoio constituída por irmãs, avós e maridos. Em outros, os filhos ficam sob os cuidados de pessoas que não fazem parte do círculo familiar, o que as deixa inseguras.

[...] eu não estava esperando ficar longe do meu filho, e me pegou de surpresa [...]. (P9) 
[...] Eu tenho um neném de três anos e ele fica com minha filha de 12, aí fica ruim é a primeira vez que eu fico longe dele [...]. (P10)

[...] Eu tenho que deixar a minha filha com alguém, esta tem sido minha maior preocupação até hoje [...]. (P9)

\section{DISCUSSÃO}

O rigor de um estudo qualitativo pode ser estimado de várias maneiras. Na presente investigação utilizou-se o critério da credibilidade ao se proceder a validação dos dados com as participantes e as enfermeiras que prestavam cuidados na clínica obstétrica. Para tanto, as colocações feitas por ocasião das entrevistas foram lidas pelas gestantes para que pudessem confirmar ou modificar o conteúdo obtido, bem como pelas enfermeiras que, baseadas na experiência prática, opinaram sobre a pertinência dos discursos. Além disso, o critério de confirmabilidade também foi considerado, visto que a enfermeira responsável pela coleta de dados tinha experiência na assistência de enfermagem a mulheres internadas com DMG. Assim, os achados puderam ser trabalhados criteriosamente, de modo que fossem posteriormente confirmados ${ }^{(15)}$.

As mulheres expressaram suas percepções e sentimentos acerca do DMG por meio de linguagem verbal e de desenhos. $\mathrm{O}$ desejo de ser mãe sobrepôs-se à gestação de risco, apesar do potencial ameaçador da gravidez com DMG. A vontade de ter um filho muitas vezes supera o medo de enfrentar complicações no desenrolar da gravidez ${ }^{(6,16)}$. Contudo, é necessário considerar fatores tais como história pessoal da gestante, idade, seu passado obstétrico, contexto da gravidez e a qualidade do vínculo com o parceiro, para compreender as reações dessas mulheres e suas vivências do ciclo gravídico-puerperal, considerando sua complexidade ${ }^{(16)}$. A experiência vivida em uma gravidez com DMG pode resultar em mudança de atitude, do estilo de vida e em motivação para realizar o autocuidado na tentativa de preservar a saúde e a vida do binômio mãe-filho ${ }^{(17)}$.

Embora a hospitalização seja necessária para o controle do DMG, nem sempre as mulheres conseguem ter acesso a um serviço especializado, com os recursos humanos e o suporte material necessários. Sobre esse fato, estudiosos evidenciam que adoecer não é uma experiência fácil, ainda mais quando incide na gravidez e é preciso utilizar um serviço de saúde pública no Brasil. A desorganização da rede de serviços de saúde contribui para a produção de doença, gerando outros sofrimentos, além daqueles que a enfermidade inevitavelmente determina ${ }^{(18)}$.

Durante a hospitalização as gestantes compartilharam entre si - e com os profissionais - ansiedades, medos, expectativas e alegrias. Perceberam que ouvindo e convivendo com as demais pacientes da enfermaria, a experiência do DMG torna-se menos caótica e ameaçadora ${ }^{(19)}$. Esse achado mostra que a existência humana é sempre ser-Com-O-outro. Na perspectiva fenomenológica, o relacionamento evidenciado com os pares e com a equipe de saúde representa um encontro de subjetividades, que promove a intersubjetividade.

O mundo no qual o homem existe não está restrito ao espaço geográfico, mas à construção humana que ele realiza para viver junto aos outros, referindo-se sempre a uma rede de relações significativas. É preciso compreender que ser-no-mundo é ser-com-os-outros, isto é, que o que somos decorre do modo como nos relacionamos e vivemos com os nossos semelhantes. O ser humano não é sozinho no seu pensar, sentir e existir; ele co-habita um mundo que passa a ser significativo para ele $^{(14)}$. "O que é vivido pelos outros nos aproxima, nos torna parceiros e, por isso, para compreendermos a experiência do outro, é necessário estarmos-com-ele, juntos-dele, para descobrirmos o ser que se oculta"(20).

A prática clínica mostra que comunicar o diagnóstico de DMG constitui uma situação delicada para os profissionais de saúde e, sobretudo, para as gestantes, por acarretar choque e desestabilização emocional. Dados obtidos em estudo anterior mostraram que, diante do diagnóstico de DMG, as mulheres relatam medo, choque e ansiedade ${ }^{(17)}$.

A descrição das experiências das mulheres com DMG trouxe à luz repercussões relativas ao distanciamento do ambiente familiar, à necessidade de redistribuição de papéis e funções na família, além de preocupações relativas aos cuidados com outros familiares. $\mathrm{O}$ adoecer gera impactos na vida prática e promove mudanças na rotina, levando à ruptura da realidade cotidiana. Planos são adiados e atividades são sumariamente interrompidas, exigindo um esforço adaptativo para o qual a pessoa tem de mobilizar uma parcela substancial de suas reservas de energia ${ }^{(18)}$.

Devido a essas repercussões, é importante que os profissionais de saúde, sobretudo os enfermeiros, estejam genuinamente abertos para potencializar o diálogo com as pacientes no cotidiano hospitalar. É preciso acolher os sentimentos contraditórios despertados pelo diagnóstico, que também se misturam às histórias de vida e aos resultados de experiências anteriores de lidar com adversidades. Experiências compartiIhadas com outras gestantes que vivenciam condições similares permitem apreender que as situações adversas que as pacientes necessitam enfrentar tornam-se mais tranquilas e aceitáveis diante do encontro autêntico com outro ser, que esteja aberto e presente para auxiliá-las.

Este estudo fenomenológico, ao possibilitar apreender a experiência das mulheres com DMG, contribui para o planejamento e a implementação de programas de intervenção baseados em um modelo de saúde participativo e que priorize a escuta da voz do outro e até mesmo seu silêncio, gestos e outras expressões da linguagem não verbal, com vistas a valorizar os aspectos subjetivos envolvidos na gravidez de risco.

A presente investigação apresenta algumas limitações, como o fato de os dados não serem passíveis de generalização. Recomenda-se que esse desenho de estudo seja reproduzido em outras regiões e países com culturas diferentes, a fim de conhecer os significados que permeiam a experiência do DMG, para ampliar a compreensão do fenômeno pesquisado.

\section{CONSIDERAÇÕES FINAIS}

Compreender o significado do DMG no cotidiano das muIheres estudadas permitiu explorar, descobrir e entender suas ideias e sentimentos. Dar voz às pessoas que vivenciam de 
maneira singular essa doença é importante para a organização do cuidado. O conhecimento produzido favorece a redução da ansiedade e a melhoria da qualidade de vida dessas mulheres, humanizando os espaços de saúde.

Na perspectiva das pacientes, ter DMG significa vivenciar experiências que trazem felicidade e sofrimento. Esse dualismo perpassa todos os discursos produzidos e constitui a essência do fenômeno investigado no presente estudo. No primeiro caso, predominam o prazer de gestar e de ser mãe, a sensação de felicidade conquistada com a oportunidade de realizar o tratamento e a esperança de obter a cura da doença após o parto, bem como a possibilidade de ser-com-o-outro durante a hospitalização. No segundo caso, o sofrimento é suscitado pelo impacto do diagnóstico, resultando em tristeza e preocupação, além da segregação dos familiares e afastamento do cotidiano doméstico. Essa polaridade de significados atribuídos à experiência do adoecer emergiu, no presente estudo, das formas de linguagem utilizadas pelas participantes, que permitiram alcançar a essência do fenômeno, desvelando toda riqueza, ambiguidade e complexidade de ser gestante com DMG.
No contexto do cuidado, torna-se imprescindível considerar essas mulheres em sua totalidade, e não apenas supervisionar os riscos associados à condição. Trata-se de uma situação extremamente complexa, tanto do ponto de vista individual como social, que se estende à esfera familiar e do casal. A mulher, ao atravessar essa experiência, enfrenta uma vivência paradoxal, pois, de um lado, está preparando e sustentando uma nova vida e, de outro, sente-se ameaçada por forças que põem em risco o ser que está gestando em seu ventre.

Nessa situação crítica, vários fatores combinam-se e interagem de forma complexa, como a história pessoal, os antecedentes gineco-obstétricos da mulher, a aceitação da gravidez e do DMG, as condições socioeconômicas, culturais e emocionais, além do acesso a serviços especializados e a qualidade do cuidado prestado. Os profissionais de enfermagem precisam estar sensibilizados e preparados para a delicada tarefa de cuidar dessas gestantes e suas famílias, criando possibilidades de acolhimento que contemplem a totalidade do ser humano, de modo a favorecer a integralidade do cuidado.

\section{REFERÊNCIAS}

1. Sociedade Brasileira de Diabetes . Diretrizes da Sociedade Brasileira de Diabetes. São Paulo; 2011.

2. World Health Organization. Definition, diagnosis and classification of diabetes mellitus and its complication: report of a WHO Consultation. Geneva: WHO; 1999.

3. Sociedade Brasileira de Endocrinologia e Metabologia. Diabetes mellitus gestacional. Rev Assoc Med Bras 2008;54(6):477-80.

4. Getahun D, Nath C, Ananth CV, Chavez MR, Smulian JC. Gestational diabetes in the United States: temporal trends 1989 through 2004. Am J Obstet Gynecol 2008;198(5):525.e1-e5.

5. Nolan JA, McCrone S, Chertok IR. The maternal experience of having diabetes in pregnancy. J Am Acad Nurse Pract 2011;23(11):611-18.

6. Silva L, Santos RC, Parada CMGL. Compreendendo o significado da gestação para grávidas diabéticas. Rev Latino-Am Enferm 2004;12(6):899-904.

7. The Hapo Study Cooperative Research Group. Hyperglycemia and adverse pregnancy outcomes (HAPO). N Engl J Med 2008;358:1991-2002.

8. International Association of Diabetes and Pregnancy Study Group recommendations on diagnosis and classification of hyperglycemia in pregnancy. Diabetes Care 2012;33:676-82.

9. Marcus MT, Liehr PR. Abordagens de pesquisa qualitativa. In: Lobiondo-Wood G, Harber J. Pesquisa em enfermagem: métodos, avaliação crítica e utilização. 4. ed. Rio de Janeiro: Guanabara Koogan; 2001. p. 122-37.

10. Spielberg HJ. Doing phenomenology. Dordrecht, Netherlands: Martinus Nijhoff; 1975.
11. Valladares ACA. Manejo arteterapêutico no pré-operatório em pediatria. Rev Eletronica Enferm [periódico na internet]. 2004 [acesso em 30 mar 2012];6(1):110-15. Disponível em: http://www.fen.ufg.br/fen revista/revista6 1/ r3_arteterapia.pdf

12. Polit D, Beck $C T$, Hungler BP. Fundamentos de pesquisa em enfermagem: métodos, avaliação e utilização. 5. ed. Porto Alegre: Artmed; 2004.

13. Colaizzi PF. Psychological research as the phenomenologist view it. In: Valle RS, King M, editors. Existential phenomenological alternatives for psychology. New York: Oxford University Press; 1978. p. 48-71.

14. Heidegger M. Ser e tempo. 3.ed. Petrópolis: Vozes; 2000.

15. Sandelowski $M$. The problem of rigor in qualitative research. Adv Nurs Sci 1986;8(3):27-37.

16. Dourado VG, Pelloso SM. Gravidez de alto risco: o desejo e a programação de uma gestação. Acta Paul Enferm 2007;20(1):69-74.

17. Evans MK, $\mathrm{O}^{\prime}$ Brien B. Gestational diabetes: the meaning of an at-risk pregnancy. Qual Health Res 2005;15(1):66-81.

18. Moreira V, Nogueira FNN, Rocha MAS. Leitura fenomenológica mundana do adoecer em pacientes do serviço de fisioterapia do Núcleo de Atenção Médica Integrada da Universidade de Fortaleza. Estud Psicol 2007;24(2):191-203.

19. Dias DG, Santana MG, Santos E. Percebendo o ser humano diabético frente ao cuidado humanizado. Rev Bras Enferm 2006;59(2):168-71.

20. Armond LC, Boemer MR. Convivendo com a hospitalização do filho adolescente. Rev Latino-Am Enferm 2004;12(6):924-32. 